\title{
Les perceptions du cancer par les patients, leur entourage et les soignants à Lomé, analyse descriptive au CHU SO, à la clinique Biasa et à la clinique de l'aéroport
}

\author{
Amekoudi Ablavi Délali Germaine, \\ Département d'anthropologie et d'études africaines, \\ Université de Lomé, Togo
}

Doi:10.19044/esj.2020.v16n11p178 URL:http://dx.doi.org/10.19044/esj.2020.v16n11p178

\section{Résumé}

Cette recherche avait pour objectif d'analyser les perceptions du cancer par les malades, les membres de leur entourage et les acteurs de soin intervenant dans le traitement du cancer à Lomé. L'enquête de terrain a été principalement effectuée dans trois formations sanitaires de Lomé auprès de la population-cible. Le travail s'est basé sur une méthodologie qualitative de type ethnographique. Cette dernière a consisté en une observation participante en situations hospitalières avec des entretiens approfondis auprès des différentes catégories d'informateurs. L'étude s'inscrit dans la théorie des représentations sociales et rend compte de plusieurs résultats. Ainsi, pour les acteurs rencontrés, le cancer reste une maladie associée à la peur, à la fatalité et à la mort. Il est difficilement appréhendé par les malades et par leurs proches. On le perçoit le plus souvent comme une maladie incurable et causée par la modernité. Pour certains, le cancer résulte d'un envoutement ou d'un mauvais œil, faisant de la pathologie, une maladie spirituellement provoquée. Elle prend aussi très souvent l'image d'une maladie de l'épreuve ou de la punition suite à une transgression. Au sein même du personnel soignant, on rencontre des perceptions de la maladie qui sont similaires à celles des patients et de leurs proches. Toutefois, plusieurs acteurs de soin, notamment les médecins et les spécialistes considèrent le cancer comme étant une maladie au même titre que les autres, même si sa définition par la science reste entourée de flou.

Mots clés: Cancer, perceptions, représentations sociales, Lomé 


\title{
The Perceptions of Cancer by Patients, Their Entourage and Caregivers in Lomé, Descriptive Analysis at CHU SO, Biasa Clinic and the Airport Clinic
}

\author{
Amekoudi Ablavi Délali Germaine, \\ Département d'anthropologie et d'études africaines, \\ Université de Lomé, Togo
}

\begin{abstract}
The aim of this research was to analyze the perceptions cancer patients, their family members and healthcare professionals have of cancer as a pathology in Lomé. The field survey was mainly carried out in three healthcare centers in Lomé with a target population. The work is based on a qualitative method of an ethnographical nature. It consists of a field observation in hospital situations with an extensive interview with different categories of informants. The study is part of the theory of social representations and gives account on the various reports received. Thus, for the individuals we met, cancer remains a disease associated with fear, fatality and death. It is difficult for the sick and their loved ones to grasp. It is most often perceived as an incurable disease caused by modernity. For some, cancer results from a bewitchment or an "evil eye", making the sickness a spiritually induced disease. Often, it takes the form of a disease of divine trial or a punishment due to a transgression. Even within the healthcare workers, some perceive the disease in a way similar to that of patients and their relatives. However, most healthcare workers, especially doctors and specialists consider cancer as a disease just like other diseases.
\end{abstract}

Keywords: Cancer, perceptions, social représentations, Lomé

\section{Introduction}

Le cancer est médicalement défini comme une tumeur maligne dont l'extension peut être locale, locorégionale, c'est-à-dire ganglionnaire ou générale c'est-à-dire métastatique (Lacombe, Pradel \& Raynaud, 2005). C'est une maladie qui se caractérise par une prolifération cellulaire anormalement importante au sein d'un tissu normal de l'organisme de telle manière que la survie de ce dernier est menacée. Sur le plan social, le cancer est entouré de 
plusieurs incertitudes tant en ce qui concerne le domaine de la biomédecine que dans la compréhension des individus (Lebeer, 2016). En effet, les causes de la maladie sont indéterminées et les traitements ne peuvent pas toujours garantir la bonne santé ou la guérison du malade (Dany, 2011 ; Harouna, 2001). On ne parle que de rémission, car la maladie peut resurgir à tout moment. Cette situation accentue et rend complexes les perceptions socioculturelles sur la maladie, aussi bien dans le corps médical que dans l'imaginaire des patients et de leur entourage. Chaque acteur construit ses subjectivités sur la maladie (Derbez, Hamarat \& Marche, 2016).

Dans l'appréhension générale des individus, lorsqu'on tombe malade, on se soigne, et, on recouvre la santé. Cependant, lorsque la santé devient difficile à retrouver ou lorsqu'il est dit qu'on ne la retrouvera plus jamais comme elle était et qu'il s'agit d'une maladie à longue durée, la maladie prend un caractère anormal (Canguilhem, 1966). Elle expose le patient et son entourage à de multiples formes de constructions, les unes aussi complexes que les autres (Reach, 2006 cité par Bourdon, 2012). Ces constructions tentent de trouver une explication à la pathologie nouvellement annoncée. Selon Kluber-Ross (1976), l'annonce d'une maladie chronique au patient lui fait subir un choc important avant d'arriver à accepter la pathologie. Ainsi, l'individu malade et ses proches cherchent à trouver les «vraies » causes de la maladie, afin de mieux la comprendre (Herzlich, 1969 ; Laplantine, 1986). Voilà ce qui se passe lorsqu'on évoque les maladies chroniques, en l'occurrence, le cancer.

$\mathrm{Au}$ Togo, les cancers les plus fréquents chez les hommes sont le cancer de la prostate et celui du poumon et chez la femme, ce sont les cancers du sein et celui du col de l'utérus (Amégbor, Darre, Ayena, Padaro, Tengue, Abalo \& Napo-Koura, 2011 ; OMS, 2014,). Dans l'espace urbain de Lomé, ainsi que dans les différentes formations sanitaires, beaucoup de considérations circulent sur le cancer, faisant état d'un manque d'information et de communication sur la maladie (Gnamien, 2018). Les patients, leurs proches et plusieurs acteurs de soin redoutent la maladie. Les incertitudes sur ses causes, ses traitements et sur les possibilités de rémission animent plusieurs conversations et sont même l'objet de beaucoup d'informations sur les réseaux sociaux (Kpotchou, 2018). Ce constat suscite un questionnement sur les perceptions que les différents acteurs directement touchés par la maladie ont du cancer en tant que maladie chronique.

À cet effet, comment les acteurs de soin, les patients et leurs proches perçoivent-ils le cancer à Lomé ? Cette question suscite des interrogations spécifiques qui se présentent comme suit : que pensent les acteurs de soin du cancer ? Comment les malades et leurs proches comprennent-ils le cancer en tant que maladie ? Pour tenter de répondre à ces questions, ce travail se donne 
pour objectif d'analyser les perceptions que les acteurs de soin, les patients et leurs proches ont du cancer.

\section{Cadre théorique d'analyse}

Ce travail s'est inscrit dans la théorie de la représentation sociale de Moscovicci (1969). En effet, selon Moscovicci, la représentation sociale est une forme de connaissance, socialement élaborée et partagée, ayant une visée pratique et concourante à la construction d'une réalité commune à un ensemble social (Jodelet, 1984). Ainsi définie, la représentation sociale est une construction qu'un individu et son entourage se font d'une réalité, d'un objet ou d'une situation. C'est un ensemble d'opinions, de croyances, d'informations individuelles ou collectives qui sont partagées sur la réalité ou la situation en question. Elle est d'autant plus importante lorsqu'il s'agit de santé et de maladie. Tout événement important de l'existence humaine exige une explication, il en va de même pour la maladie, dont on doit trouver la nature, les causes, les implications et des explications à même de rendre celleci intelligible et compréhensive à la personne ou au groupe concerné. Cependant, l'élaboration des causes d'une maladie n'est pas seulement individuelle, mais également reliée au social et au culturel et surtout interprétée en fonction des contextes sociaux dans lesquels ils se produisent (Herzlich, 1969). La maladie est considérée comme un fait profondément social et ne peut être abordée sans prendre en compte son rapport avec les individus et leur société (Laplantine, 1986). Augé (1984), pour sa part considère, de manière anthropologique, la maladie comme un désordre biologique et social soulignant ainsi le caractère social que peut revêtir une maladie.

Dans le cadre de ce travail, cette théorie a permis d'analyser les représentations socioculturelles que les acteurs de soin, les malades et leurs proches ont du cancer et les constructions sociales qui l'entourent. En effet les acteurs de soin, les patients et leurs proches construisent socialement la réalité de la maladie qu'est le cancer, et ce sont ces constructions qui orientent leurs perceptions de la maladie.

\section{Méthodologie}

\section{La zone de recherche}

Cette recherche a été effectuée dans la région sanitaire Lomécommune qui est l'une des 6 régions sanitaires du Togo. La ville de Lomé est la capitale du Togo. Elle constitue un grand carrefour socioculturel et économique où se côtoient des peuples venus d'horizons divers (Ourobitasse, 2015). Cependant, les peuples autochtones de Lomé sont des éwés, un des peuples issus de l'aire culturelle Adjatado qui s'étend dans presque tout le sud du Togo (Gahibor, 2011). La langue locale la plus parlée à Lomé est l'éwé. 
Chez les éwés du Togo, la maladie est un problème profondément social (Atchrimi, 2008).

La région sanitaire Lomé-Commune est l'une des régions les plus importantes du Togo en matière d'institutions et de structures de santé. Elle abrite toutes les institutions centrales en matière de santé dans le pays. Lomé dispose également de deux Centres Hospitaliers Universitaires (CHU) sur les trois disponibles au Togo. Ce sont le CHU Sylvanus Olympio qui est considéré comme la première formation sanitaire de référence du pays et le CHU Campus qui est la deuxième. C'est au CHU SO que sont pris en charge la grande partie des malades de cancer au niveau public dans tout le pays. Le centre abrite également le laboratoire d'anatomopathologie. Dans le cadre de cette recherche, deux services du CHU SO ont été touchés. Il s'agit de la clinique médico-chirurgicale communément appelée « Pavillon Militaire », et de la clinique de gynécologie, notamment son étage $B$, habituellement désigné «Gynéco B ». Ces deux unités contiennent en leur sein des équipes de soins oncologiques. Au Gynéco B seuls les cancers de type gynécologiques sont pris en charge, donc uniquement des femmes, et au Pavillon militaire, tous les types de cancers sont pris en charge. Lomé compte également plusieurs formations sanitaires privées de référence qui prennent en charge les cancers, notamment la clinique de l'aéroport et la clinique Biasa, qui sont les deux formations sanitaires privées où a été menée cette recherche.

\section{Démarche}

Ce travail est basé sur une méthodologie essentiellement qualitative de type ethnographique. Le choix de cette démarche méthodologique s'explique par son importance dans l'appréciation des données à collecter. Il s'agit ici de décrire et d'analyser les perceptions du cancer par les malades, leur entourage et les soignants. Cette méthodologie s'est basée sur deux principales techniques, les observations et les entretiens. Le choix de la technique de l'observation s'explique par le fait qu'elle assure une double démarche d'élaboration de savoirs en aidant à la fois, à répondre à des questions sur l'objet de recherche et à analyser la façon dont on procède pour choisir cellesci, mais aussi à élaborer une stratégie (Blanchet Ghiglione, Massonat \& Trognon, 2005). L'entretien pour sa part a permis de recueillir des informations sur les perceptions des différents acteurs. Il est considéré comme la technique la plus évidente en sciences sociales (Blanchet \& al, op. cit.). Ainsi, une grille d'observation et des guides d'entretiens ont été élaborés pour la collecte des données lors de l'enquête.

La grille d'observation est élaborée avec l'objectif d'observer en situation de soins les différents acteurs. Les observations ont été faites dans différentes situations, notamment en salles d'attente, lors des consultations, lors de séances de chimiothérapie, dans les salles d'hospitalisation pour les 
patients qui nécessitent un soin permanent ou sous soins palliatifs. Il s'est agi d'assister à différentes scènes d'interactions entre les soignants, les patients et les membres de leur entourage, à suivre leurs différents échanges et conversations et au même moment à poser des questions lorsque la situation le permet. Dans les conversations on peut déjà relever différentes perceptions de la maladie par les individus en relation. Les observations ont été permanentes pendant toute la durée de l'enquête de terrain.

Les entretiens ont été faits de manière semi directive. Le guide d'entretien est élaboré autour de questions concernant ce que les différents acteurs pensent de la maladie, de ses causes et de ses implications et comment ils comprennent celle-ci. Lors de la collecte de données, une phase a été consacrée uniquement aux observations en vue de se familiariser avec les acteurs à enquêter et les différentes pratiques en oncologie. Une deuxième phase a couplé l'entretien à l'observation. En effet, la plupart des entretiens avec les patients et les membres de leur entourage ont été effectués en salle de chimiothérapie principalement pour les cliniques privées ou en salle d'hospitalisation fondamentalement pour le Gynéco. D'autres patients ont été enquêtés lorsqu'ils étaient en salle d'attente (Gynéco B et Pavillon Militaire). Ainsi, une salle libre a été mise à disposition pour pouvoir les questionner à l'abri des regards. Les soignants sont généralement interrogés avant et après les consultations et la chimiothérapie et les contrôles de routine dans les salles d'hospitalisation ou à des heures creuses. Cependant, avec les soignants, beaucoup d'entretien ou de questions ont été suscités par des faits ou des situations observées. Dans ce cas, ils sont interrogés juste ou peu après les faits observés lorsque la situation le permet, c'est-à-dire lorsque le patient se retire ou lorsque le soignant lui-même quitte le patient pour continuer seul ses activités. Les entretiens se sont principalement déroulés en français ou en éwé selon le choix du patient et selon la langue dans laquelle ce dernier se sent plus à l'aise. Parfois, les deux langues interviennent dans les entretiens. Avec les soignants cependant, les entretiens ont été le plus souvent faits en français, langue qui donnait plus de facilité à ces derniers de s'exprimer sur le cancer selon les observations faites lors de leurs interactions avec les malades.

En ce qui concerne la durée des entretiens, avec les soignants, elle est indéterminée parce que chaque fait rencontré peut faire objet d'un entretien approfondi. Avec les patients reçus en salle isolée au Gynéco B notamment, les entretiens ont duré entre 25 et 45 minutes, selon la nature des données recueillies, la collaboration et la patience du patient et selon le nombre de patient présent en salle d'attente. Ces entretiens se sont déroulés de manière plus ou moins formelle. Cependant, dans les salles de chimiothérapie et d'hospitalisation, lorsque le patient le permet, la durée des entretiens est également indéterminée et dépend de la richesse des données à fournir et de l'implication du patient. En effet, ces entretiens ont été pour la plupart non 
formels. Après introduction et explication de l'objectif de l'entretien au patient et après que ce dernier a signé la fiche de consentement, l'entretien est abordé comme une conversation une discussion comme une autre avec le patient et dans certains cas son accompagnant. Ce genre d'entretien dans le cadre de ce travail a permis de recueillir des données plus riches parce que le patient n'a pas l'impression d'être interrogé mais de converser avec quelqu'un pendant qu'il est sous chimiothérapie. Il faut noter que certains patients peuvent passer jusqu'à trois voire quatre heures sous chimiothérapie. Tout comme avec les soignants, avec plusieurs patients les entretiens se sont déroulés en plusieurs séances selon les rendez-vous obtenus pour le suivi des consultations et pour la chimiothérapie. Il en a été de même avec les patients hospitalisés ou sous soins palliatifs, étant donné qu'ils sont présents pour une durée assez longue, les entretiens se sont échelonnés sur plusieurs jours permettant ainsi aux patients de ne pas s'ennuyer des questions et de donner des informations plus approfondies sur des sujets déjà discutés.

$\mathrm{Vu}$ le caractère sensible de la thématique du cancer, étant donné que plusieurs patients dès le début de l'enquête ont décliné l'utilisation de l'enregistreur ou ont été hésitants quant à l'utilisation de cet appareil, les entretiens n'ont pas été enregistrés. Ceci en vue de garantir aux patients l'anonymat et le respect de leur intimité. Les données sont notées au fur et à mesure que les entretiens évoluent.

Des entretiens de groupe ont également été organisés dans les salles communes de chimiothérapie. Les effectifs des personnes interrogées ont varié entre 2 et 6 personnes. Par ailleurs, deux fora de discussions organisés par des associations ont également permis de recueillir des données sur les perceptions de la maladie. Au cours des fora de discussion, le nombre des enquêtés a varié entre 10 et 20 patients et membres de famille. La collecte de données dans les formations sanitaires et dans les différents espaces de soins a été possible grâce à une autorisation reçue du Comité Bioéthique pour la Recherche en Santé (CBRS) et à l'autorisation du Ministère en charge de la santé. Ces autorisations ont été validées par les chefs services et les médecins traitants qui ont permis d'avoir accès à leurs patients et à leurs services.

Par ailleurs, des entretiens ont également été organisés avec des personnes-ressources du Ministère de la Santé et de la Protection Sociale, des acteurs d'associations qui ont permis d'avoir accès à quelques patients à domicile, et des personnes-ressources de la culture éwé. Après la collecte et la saturation des données, l'effectif des enquêtés fait état de 41 hommes et femmes malades de cancer ; 16 membres de l'entourage, 26 soignants et 20 personnes-ressources.

Le groupe cible visé par la recherche est composé des patients de cancer, de leur entourage et de leurs soignants. L'étude s'est concentrée en milieu hospitalier, car hors des formations sanitaires, il était difficile de 
rencontrer des malades de cancer à leurs domiciles. Elle a donc visé les patients, les membres de leur entourage, notamment les accompagnateurs, et le corps soignant. Par corps soignants, sont désignés tous les acteurs de soin qui interviennent dans le traitement des patients ou du malade. Ici, la notion de soignant n'est pas limitée à la fonction technique du médecin qui agit sur la santé de l'individu. Cette notion regroupe tous les acteurs qui interviennent dans le soin au malade, qui font partie d'une l'équipe pluridisciplinaire, qui ont tous pour mission fondamentale de prendre soin des personnes malades, quelle que soit la spécificité de leur métier (Hesben, 2007 cité par Schwartz, 2011). Il s'agit donc des médecins, des sages-femmes, des infirmiers et des garde-malades qui travaillent dans les services étudiés et qui entretiennent des liens de soins avec les malades.

Les données analysées dans ce travail ont été collectées sur une période de 16 mois discontinue, allant de janvier 2016 à juillet 2019. Les données recueillies à travers les notes d'observation et d'entretien ont été transcrites de façon manuelle à la fin de chaque journée de collecte de données. Après cela, elles ont été triées et recoupées par thématique selon les objectifs de la recherche, à savoir les perceptions que les soignants ont du cancer et les perceptions que les patients et les membres de leur entourage ou leurs accompagnateurs ont de cette maladie. Les méthodes d'analyse du contenu (Bardin, 1983) ont permis de faire l'analyse des données. Ce sont d'une part, l'analyse thématique catégorielle qui consiste en la comparaison de la fréquence de certaines caractéristiques ou de certains thèmes importants en lien avec les objectifs de la recherche, évoqués par le locuteur. D’autre part, l'analyse formelle de l'énonciation a permis en prêtant attention aux données telles que l'ordre des mots, les répétitions et les ruptures de ressortir les différentes énonciations en lien avec les objectifs de l'étude. Après cela, les principales tendances enregistrées ont permis d'arriver aux résultats présentés ci-dessous.

\section{Résultats de la recherche}

\section{Le cancer, d'une maladie chronique à une maladie " sans tête »}

Les populations togolaises ont une connaissance des maladies chroniques. Au sud-Togo par exemple, elles sont désignées sous le vocable dole mavo : une contraction de dolele (maladie) et de mavs (infinie), ce qui veut dire au sens littéraire, une maladie qui ne finit pas. Cette appellation concorde avec la définition de l'Organisation Mondiale de la Santé (OMS) qui dit des maladies chroniques qu'elles sont des problèmes de santé nécessitant des soins à long terme (OMS, 2015). Cependant, parmi les maladies chroniques, il y en a que la communauté éwé considère comme comprises à l'instar de la tuberculose (yəme $k p \dot{\varepsilon}$ ). En effet yəme signifie au sens littéral pays des morts et $k p \dot{\varepsilon}$ veut dire toux. Dans son sens littéraire, la maladie veut 
donc dire « la toux qui conduit à la mort ». Cette interprétation de la maladie la présente clairement comme une maladie qui ne finit pas et qu'on ne peut pas guérir. En effet, même si aujourd'hui la tuberculose a des traitements efficaces et peut être guérie, la communauté éwé a pendant longtemps cerné et partagé son caractère chronique d'autrefois. Cependant dans le registre des maladies en éwé, il existe certaines maladies chroniques dont la compréhension échappe à la communauté. Elles sont considérées comme n'ayant aucune explication logique, car on n'en connait ni les causes, ni les traitements adéquats et sûrs, et on ne peut pas les guérir. Ces maladies sont nommées «domanya ta na» (maladie sans tête au sens littéral et maladies bizarres, maladies incompréhensibles au sens littéraire). Parmi ces maladies qu'on ne comprend pas en pays éwé on peut compter le cancer. C'est ce qu'on peut lire à travers les propos suivants recueillis auprès des acteurs rencontrés. "On nous dit que la personne a un cancer, que nous devons faire la chimiothérapie, que même si la chimio l'abat, elle pourra réduire la maladie, mais depuis là on nous prescrit les médicaments mais la maladie revient toujours, on n'y comprend rien » (parent de patient en métastase osseuse d'un cancer du sein, octobre 2018).

«Ce n'est pas une maladie que vous devez chercher à comprendre, nousmême on n'y comprend rien, on ne sait pas ce qui en est véritablement la cause, on en connaît que les facteurs de risques, et encore, ce n'est pas parce qu'on est exposé aux facteurs de risques qu'on l'a automatiquement. La question du cancer, c'est que, si tu ne l'as pas, il faut remercier Dieu et prier pour ne jamais l'avoir, si tu l'as, il faut prier pour avoir les moyens de suivre les traitements qu'il faut et il faut espérer que ton organisme réponde bien, savoir que ce n'est pas de ta faute, que tu ne peux rien faire contre, tu ne peux faire que ce que tu peux et ce que la médecine peut » (Réponse d'un médecin à un homme souffrant de cancer de sein qui a demandé à savoir la cause exacte de la maladie, novembre 2018).

Comme on peut le voir à travers les propos ci-dessus, le cancer est sujet à beaucoup d'incompréhensions. D'une part à cause de l'absence d'informations scientifiques voulues ou souhaitées sur la maladie et d'autre part à cause de l'incertitude et de la peur dans laquelle elle met patients, entourage et soignants. Le cancer est considéré comme une maladie de l'incertitude, à cause des contours flous de ses causes et de ses possibilités de guérison. Elle n'est pas ainsi considérée uniquement chez les malades ou chez les membres de leur entourage, mais aussi par les acteurs du personnel soignant. En effet, même en milieu hospitalier, on peut entendre des discours d'incertitude et d'incompréhension sur la maladie. Les sages-femmes et les médecins rencontrés ont exprimé leur peur vis-à-vis de cette maladie que «personnes ne comprend». Le flou biomédical autour des causes du cancer et des possibilités de rémission accentue encore plus cette perception dans 
l'imaginaire des individus. Souvent les médecins spécialistes tentent de l'expliquer aux patients et à l'entourage; mais la définition même de la maladie semble encore plus flou pour certains, car la cause de cette «multiplication anarchique des cellules » n'est jamais claire et spécifique. Ainsi, lorsque le soignant spécialisé lui-même a du mal à parler de la cause d'une maladie, l'ambiguité autour de la pathologie prend une dimension beaucoup plus complexe.

C'est peut-être cette ambiguïté qui fait que le cancer n'a pas de dénomination fixe connue en langues locale au sud Togo. La nomination des maladies considérées comme "nouvelles » à la culture est en général des néologismes basés sur la description médicale ou les manifestations observables de la maladie (Bonnet \& Jaffré, 2003 ; Jaffré, 1999). Cependant, dans le cas du cancer, la maladie est généralement désignée "cancer » et prononcée comme en anglais. Les autres dénominations que nous avons pu observer sur le terrain ne sont pas unanimes, mais expriment toutes la peur, le danger et l'incertitude autour du cancer. On peut noter $d s$ bada $(d s=$ maladie ; $b a d a=$ mauvaise, méchante, dangereuse $) ; d o d z i n(d o=$ maladie $; d z$ in $=$ rouge , danger, feu) ou encore abi makou makou (abi = plaie; makou makou =inguérissable, infinie). Les éléments à retenir dans ces appellations sont les adjectifs qui qualifient la maladie et la connotation qu'ils ont en général dans la communauté. Le rouge exprime le danger, le sang, le feu, le mal, et les autres adjectifs font état de l'incertitude de la maladie. Ainsi, la construction sociale autour du cancer est d'abord émaillée de négativité et d'incertitudes. Cependant les constructions autour du cancer ne s'arrêtent pas là.

\section{Le cancer, une maladie des blancs}

Le cancer est considéré comme «une maladie des blancs», une maladie des pays riches ou de l'occident. Cependant, cette considération a un double sens. Le premier sens est lié à la considération de la maladie comme une maladie étrangère. Et l'étranger duquel viendrait cette maladie est l'occident. Ainsi, "C'est au contact des colons que le mot cancer serait apparu dans les cultures togolaises et avec lui une maladie»(Personneressource éwé, octobre 2018). Le cancer est donc considéré comme n'existant pas autrefois dans les cultures locales. Comme on peut le déduire du propos de patients rencontrés :

"Cette maladie on en entendait parler, mais on savait que c'est une maladie qui touche surtout les blancs, quand nous, on était jeunes et qu'on entend parler de cancer, d'abord les gens ne savaient pas ce que ça voulait dire et il n'y a que quelques-uns qui savent que c'est une maladie qui tue... En grandissant j'ai connu quelques personnes qui ont été atteintes de ça, mais c'est vraiment rare, aujourd'hui on l'attrape comme les autres maladies, c'est 
devenu notre maladie aussi. (Femme souffrant d'un cancer du sein, 65 ans, octobre 2018)

"Cancer, cancer, humm, nous nos grands-parents ont vécu des années et des années et sont morts devant nous très vieux, ils n'ont jamais eu aucun de ces maux, c'est chez les blancs qu'on entend ces choses-là, ou bien chez nos parents qui ont vécu là-bas. Et voilà qu'aujourd'hui nous, on a ça. » (Malade homme 70 ans, cancer de l'estomac, octobre 2018)

Comme on peut le lire dans ces propos, le cancer ne serait pas à l'origine une maladie que les Africains attrapent. Ce serait une maladie née du contact des blancs, peut être ramenée par les Africains ayant vécu en occident. La particularité avec cette perception de la maladie est qu'elle est souvent revenue dans les entretiens avec les malades, les membres de l'entourage d'âge mûres ou du troisième âge. Des personnes-ressources âgées rencontrées l'ont également évoqué. Cependant, il est à relever que c'est une perception qui n'a pas vraiment été ressortie lors des entretiens avec le personnel soignant.

En second lieu, le cancer est considéré comme une maladie des blancs en raison des possibilités de traitements disponibles ou indisponibles en Afrique, au Togo plus précisément. En effet, Cette considération est en étroit lien avec la première. "Une maladie venue des blancs ne peut être mieux traitée que chez les blancs ». Pour beaucoup, le cancer est une maladie des blancs parce que, ce sont «eux qui l'ont découvert» et ce sont donc «eux qui peuvent vraiment bien le traiter $»$. Ainsi, pour plusieurs acteurs rencontrés, malades, entourage, personnel soignant et personnes-ressources, le cancer ne peut être efficacement traité qu'en occident. Cette perception a été longtemps entretenue en raison de l'absence de spécialistes du cancer dans le pays dans un passé encore récent. Aujourd'hui, au Togo, lorsque la maladie est diagnostiquée tôt, les possibilités de traitement et de rémission sont bien reconnues.

\section{Le cancer, une maladie des riches}

La considération du cancer comme une maladie de riches n'est pas très éloignée de la précédente. Comme pour la perception précédente, celle-ci revêt également deux volets qui seront détaillés dans les propos à suivre.

"Cette maladie-là, ce n'est pas tout le monde qui l'attrape, généralement ce sont les gens qui ont beaucoup d'argent et qui mangent des choses trop riches, qui ont un certain train de vie qui ont cette maladie. Je ne sais pas pourquoi maintenant les pauvres comme nous doivent aussi faire face à cette maladie » (Instituteur, 55ans, cancer du côlon, octobre 2018).

"Avant, quand tu entends parler du cancer, tu sais que non seulement c'est un vieux ou une vieille mais aussi et surtout une personne qui sort d'une 
famille assez aisée, ce sont eux qui ont normalement cette maladie. » (Femme, 25 ans, cancer du sein, août 2016).

D’une part, le cancer est considéré comme une maladie de riches, car il serait plus fréquent chez les personnes riches que chez les pauvres selon les données recueillies. L'indicateur qui permet ici de considérer une personne comme riche est celui surtout de son niveau de vie et de son alimentation. Ainsi, les personnes consommant des nourritures trop riches, ou les familles ayant un train de vie luxueux et extravagant sont considérées comme les plus sujettes à la maladie du cancer.

D'autre part, le cancer est considéré comme une maladie des riches à cause des «coûts exorbitants» dans lesquels s'engagent les familles des patients qui en sont atteints. Comme le dit clairement une femme souffrante du cancer de sein rencontrée en août 2016, "Dès qu'on te l'annonce, tu n'y peux rien, il faut chercher de l'argent ». C'est de la portée des frais à engager que parle également une personne-ressource du ministère de la santé lorsqu'elle déclare, "Le cancer expose les malades à des coûts exorbitants, même pour les plus aisés ». Si même les personnes les plus aisées ont du mal à supporter les frais de traitement du cancer, il est important de se demander ce que cela serait pour les moins nanties. C'est fort de ce questionnement que les propos suivants viennent en illustration à la situation vécue par les malades et leurs familles dans la prise en charge du cancer. "Ce qui tue les patients, ce n'est pas la maladie elle-même mais plutôt le manque de moyens » (médecin, janvier 2017).

«Comment puis-je espérer qu'une femme qui travaille dans la zone franche, avec un mari agent de sécurité, puisse trouver les moyens de faire une radio thérapie à l'extérieur et de faire éventuellement une chimiothérapie après, comment vont-ils pouvoir trouver ces deux millions? ». (Conversation entre deux médecins se souciant de la situation d'une femme à qui l'un vient d'annoncer un cancer de l'utérus, février 2019).

«Ma sœur, ce n'est pas que je ne peux pas me battre contre cette maladie, mais je ne peux pas me battre pour des moyens que je n'ai pas... Je n'ai presque rien, c'est sur moi que repose ma famille, et ça en plus ! humm...» (Conversation entre la femme en question dans le propos précédent et une psychologue essayant de la calmer et de l'apaiser après l'annonce de la maladie, février 2019).

«Ou est-ce que je suis allée trouver cette maladie? Ce n'est pas une maladie pour les pauvres, c'est une maladie pour ceux qui ont les moyens de s'en charger... » (Femme, 46 ans, cancer du col de l'utérus, revendeuse à l'intérieur du pays, août 2016).

En effet, le cancer est une maladie qui fait beaucoup dépenser. Dans la quête de guérison, il n'est pas rare de rencontrer des familles qui ont dû vendre leurs possessions pour pouvoir prendre en charge leur malade. 


\section{Le cancer, une maladie incurable conduisant inévitablement à la mort}

Que l'on soit acteur médical, malade ou membre de l'entourage, la question des possibilités de vivre avec un cancer est la plus redoutée. Les propos ci-après viennent appuyer cette considération :

"Il y a un médecin qui m'a dit une fois, qu'il ne peut pas faire ce travail, qu'il ne peut pas travailler avec les morts, selon lui, nous prétendons soigner les gens, mais nous savons que la personne est en train de mourir. Le même médecin quand il a découvert qu'un des membres de sa famille a eu un cancer, alors que c'est lui qui traitait la personne et qui avait demandé les analyses, a mis les résultats dans une enveloppe et a dit à la personne que ce n'est pas sa spécialité et d'aller voir un autre médecin qui pourra mieux interpréter les résultats. Il avait tellement peur d'annoncer le cancer à son proche... De lui annoncer qu'il va mourir » (acteur du personnel médical, novembre 2018) "Se savoir mourant est la première image qui traverse la tête des patients lorsqu'on leur annonce la maladie » (Médecin, août 2016).

"Ma fille j'ai 60 ans, j'ai 6 enfants, j'ai vu ma propre fille mourir de cette maladie dans des souffrances atroces, quand on me l'a annoncée j'ai eu un choc, je me suis dit moi encore? Là où je suis mon médecin me dit qu'avec la masectomie je peux vivre et vaincre la maladie, mais moi je pense déjà à la mort, il n'y a pas d'autre solution pour cette maladie » (femme, 57 ans, cancer du sein, août 2016 )

"Docteur, humm vous m'avez dit que ça va me coûter beaucoup d'argent pour le traitement, je voudrais demander s'il vous plaît, est-ce que je peux au moins vivre assez longtemps pour trouver tout cet argent? 》(Une patiente juste après l'annonce de sa maladie, février 2019).

J'entends les gens parler de ça à la radio, mais je ne peux pas vous dire que je sais si ou que je sais ça sur la maladie, tout ce que je sais c'est que c'est une maladie qui tue et qui est très dangereuse, j'ai peur de mourir » (femme 60 ans, cancer de l'utérus, août 2016).

Comme le relatent ces propos, le cancer annoncé aux patients renvoie à l'idée d'une maladie sans fin et cette façon de comprendre la maladie est également présente chez les acteurs de soin. Toutefois, ce n'est pas la question de la guérison ou non du cancer qui est mise en cause ici, mais plutôt le fait que la maladie soit directement rattachée à la mort, car on peut être malade d'une maladie inguérissable sans penser à la fatalité ou à la condamnation. Porter une maladie chronique ne fait pas automatiquement du malade un article de la mort. Cependant, selon la majorité des acteurs rencontrés, le cancer est presque égal à la mort, une mort inévitable et douloureuse, voire honteuse. Une maladie dont la fatalité est presque indiscutable selon la majorité de nos répondants.

Comme évoqué un peu plus haut, les patients ne sont pas les seuls à penser que le cancer est synonyme de la mort assurée. Cette perception est 
également lue dans les propos d'acteurs du personnel médical, et même de médecins ; "Selon moi quand ça t'arrive c'est fini hein, quel que soit le moyen, quand ça arrive à un moment donné c'est fini » (Garde malade, février 2019). «Il y a certains cas, quand toi-même tu vois, tu sais que ce n'est plus la peine et quand tu leur administres des soins, tu as mal pour eux» (Sage-femme, février 2019). "Quand on était en formation et qu'on voyait un dossier sur lequel il y a écrit «NEO », on ne touchait plus, pour ces malades c'était déjà fini, on avait tellement peur d'eux » (Infirmier, novembre 2018). "Quand les collègues, médecins et infirmiers nous voient ici, ils nous demandent pourquoi c'est seulement cette branche que nous avons trouvé à embrasser dans le domaine médical, ils nous appellent les médecins des morts, ils considèrent que quelqu'un qui a un cancer a déjà un pied dans la tombe » (Infirmier octobre 2018).

"Non Docteur, je ne veux pas venir dans le service d'onco, s'il ne vous plaît pas ça, avec ce que je vis en réa, je ne peux pas supporter plus de décès. S'il vous plaît, pas ça » (un $\mathrm{DES}^{43}$ à un médecin spécialiste qui lui propose un stage dans son service d'oncologie, février 2019).

«Tu vois la jeune sage-femme là ? La fois dernière elle m'a demandé, la jeune fille qui vient chez vous elle aussi elle a choisi cette spécialité ?... Oh, pourquoi vous les jeunes vous choisissez de travailler sur le cancer? Ça ne vous fait rien de savoir que vos patients vont mourir dans très peu de temps? » (Conversation avec un infirmier, novembre 2018).

On peut également lire à travers ces propos le traumatisme vécu par les soignants dans les cas de cancer. Cette peur du cancer comme maladie conduisant inévitablement à la mort va au-delà de la maladie et est aussi rattachée aux produits intervenant dans le traitement, notamment les drogues de chimiothérapie.

«Nous avons été plusieurs infirmiers à être formés au CHU SO pour l'administration des soins oncologiques. Au cours de la formation on nous a expliqué que les produits sont très toxiques à la santé et qu'il fallait se protéger très bien pour ne pas être exposés. Juste cette explication avec ce que les gens pensent déjà de la maladie a fait fuir certains. D'autres, lorsque nous avons commencé les soins ont effectivement pris peur et ont commencé par disparaître un à un. Ils ont peur aussi de la maladie et se disent que le fait de rester avec des malades et surtout en contact avec leurs produits peut les exposer à autre chose, ce n'est pas moi qui vais dire ça...Mais moi je me dis, qu'il faut forcément quelqu'un pour s'occuper de ces malades aussi et c'est pour ça que je suis resté... »(Infirmier, février 2018). ${ }^{43}$ DES (Diplôme d'Etudes Spécialisées) est le terme par lequel sont couramment désignés les
médecins en spécialisation AU CHU SO. 
La peur des produits toxiques dont il est question est très souvent revenue lors des entretiens avec le personnel soignant y compris les médecins. Il s'agit d'une préoccupation très importante qui peut décourager à la prise en charge des patients malades de cancer. L'interpellation par un médecin spéciste sur la question lors de la collecte de données est un cas palpable. En effet, le jour-là, plusieurs patients étaient présents en salle de chimiothérapie pour leur cure, et l'environnement était propice pour une discussion en groupe. Les propos suivants sont ceux du médecin lorsqu'il a appris cela :

«Tu vas discuter avec eux pour combien de temps? Tu n'as pas peur? Non, normalement je ne devais pas te laisser entrer là-bas, moi je n'y entre jamais, c'est trop toxique. Ta blouse seule ne te suffit pas, va lui dire de te chercher une surblouse, des gants, une coiffe et une bavette. Normalement tu devais même te déshabiller et porter autre chose avant d'entrer et te rhabiller après... Tu ne dois rien ramener de tout ce qui entre dans cette salle à la maison. Tu es une femme et c'est très dangereux pour toi... Tu tiens toujours à y aller... Bon, vas-y mais pas question que tu y entres comme ça, normalement ces chaussures ne doivent plus entrer dans ta chambre à la maison... » (Médecin gynécologue, novembre 2018).

À travers ces propos, on peut percevoir toute la méfiance et la peur des acteurs de soins eux-mêmes, même les plus professionnels et les plus spécialisés à l'égard du cancer et de tout ce qui entoure la maladie.

Pour revenir à la perception de la fatalité du cancer, il convient de dire qu'elle est surtout alimentée par le nombre de décès qui survient dans la prise en charge des cas et par leur fréquence. Ceci surtout parce que la plupart des cas de cancer arrivent à l'hôpital à un stade très avancé, certains après un diagnostic trop tardif de la maladie, d'autres à cause du manque de moyens pour commencer une prise en charge à temps à l'hôpital ; et d'autres encore, après avoir parcouru d'autres formes de traitement sans réponse efficace. Un stade qui bien souvent ne donne pas d'autres possibilités aux médecins que de mettre les patients sous soins palliatifs, ou de juste essayer un traitement pour espérer avoir un résultat positif. Ainsi, plusieurs malades ne viennent à l'hôpital que « pour y mourir ». C'est-à-dire pour être mis sous soins palliatifs et bénéficier d'une surveillance permanente ou non avant de se laisser emporter par la maladie. ${ }^{44}$

\footnotetext{
${ }^{44}$ Les soins palliatifs sont des soins qui visent que le confort du malade en fin de vie. Ils ont pour but de prévenir et de soulager la douleur physique, les symptômes inconfortables comme la nausée, la constipation et l'anxiété e et la souffrance psychologique du malade. Dans plusieurs pays, les soins palliatifs sont organisés de telle sorte qu'ils incluent la famille et la religion du malade. Son but principal est de faire en sorte que les malades partent « sans avoir mal ».
} 


\section{Le cancer, une maladie spirituellement provoquée}

Selon une patiente rencontrée au CHU SO,

«Il y a une femme dans mon quartier avec laquelle je ne m'entends pas, un jour on a eu une querelle et elle m'a dit : ce n'est pas parce que tu as des pieds que tu marches pour venir me répondre? C'est vrai que ma maladie avait déjà commencé quand elle m'a dit ça, mais le soir-là même je suis passée en aller et retour devant chez elle, et c'est de retour chez moi que mon malaise qui m'a conduit ici et m'a cloué au lit a commencé. Le lendemain, je ne pouvais plus marcher. Je me demande si ce n'est pas elle qui est la cause de ma maladie et si oui, est-ce que c'était le cas depuis le début ou bien est-ce que c'est juste ce soir-là qu'elle a commencé? Parce que j'ai un cancer du sein, et tout d'un coup, alors que mon sein ne me fait plus si mal je me retrouve à ne plus marcher et à avoir mal dans toutes mes articulations, jusque dans mes os et même, à ne plus pouvoir marcher, ce n'est pas curieux ça?» (Femme en métastase osseuse d'un cancer du sein, Février 2019).

Le cancer est considéré comme une maladie physiquement et naturellement anormale par certains patients et membres de leur entourage. Un mal issu d'un mauvais œil, d'un mauvais sort, d'un envoûtement provoqué par une personne ennemie, un adversaire, souvent relevant de la famille ou du monde professionnel. La définition et la compréhension de la maladie et de ses implications sont difficilement concevables et saisissables à l'appréhension humaine. Cette situation engendre de multiples questions sur son caractère naturel. Selon les propos recueillis, une maladie aussi « grave » ne pourrait atteindre une personne sans qu'il n'y ait une mauvaise intention humaine derrière. La maladie est d'emblée placée dans un autre registre, passant de l'état de naturel à celui du surnaturel ou du spirituel. Pour un tradipraticien rencontré : «Toutes les maladies peuvent être provoquées des plus graves aux plus banales. Le cancer aussi est souvent provoqué, et quand les gens viennent chez nous, nous traitons ça spirituellement et physiquement » (août 2018).

Naturellement, à maladie physique ou naturelle, traitement naturel et à maladie surnaturelle ou spirituelle, traitement spirituel. Les propos suivants recueillis auprès des patients et des membres de leur entourage en disent plus : "Quand ma maladie a commencé, je suis allée voir plus de trois médecins qui n'ont pas pu venir à bout, c'est là que sur conseil de ma famille je suis allée voir un prêtre spirituel qui m'a consulté et il m'a dit que quelqu'un a placé quelque chose sur mon tabouret et que je me suis assise dessus. C'est ce qui me cause la maladie, et j'ai commencé le traitement chez lui. J'ai cru en ce qu'il m'a dit parce que j'étais bien portante et la maladie a surgi d'un seul coup, ce que je ne pouvais pas m'expliquer. On m'a dit que c'est un cancer, mais moi je sais que je ne souffre pas de ça, c'est Dieu seul qui sait ce que c'est. Celui qui me provoque ça aussi tant que je ne suis pas libre lui aussi il 
ne se reposera pas, mais je sais que je vais m'en sortir » (Femme cancer de la vulve, 41 ans, octobre 2018).

«Ce que je ne comprends pas, c'est comment cela peut se faire que je suis moi-même vivant mais que mon intestin soit en train de pourrir? Ce n'est pas normal ça!» (Homme, cancer du côlon, 55 ans octobre 2016).

"Humm j'ai brillamment survécu à un cancer de l'estomac et 12 ans après, alors que ma vie avait bien repris son cours, je fais une rechute, ça, c'est pour les mauvais esprits, quand on $m$ 'avait traité on $m$ 'avait dit que si ça ne revient pas dans les 5 ans, je ne crains plus rien » (66 ans, ayant suivi un traitement pour un cancer de l'estomac, et douze ans après successivement, un néo du colon, un kyste de l'ovaire et une adénopathie, octobre 2018).

«Cette maladie moi je suis sûre qu'il y a la main d'une personne dedans, non je vous assure. À chaque fois que le traitement évolue, je ne sais plus ce qui se passe et on revient en arrière. Et un autre (cancer) entre dedans. Ma fille, lui, il ne veut pas me croire, il dit que ce sont des histoires. Mais, moi sa mère, je sais que ça là ce n'est pas normal. Même le docteur a elle-même dit qu'elle ne comprend pas son cas. Quand tout ça va finir par la grâce de Dieu, il faut l'envoyer loin, loin du regard des malveillants... » (Mère d'un patient de 32 ans).

L'histoire personnelle du patient avec la maladie participe beaucoup aux perceptions de la maladie. Si nous prenons le cas de ce dernier propos, mis en relation avec l'histoire de la maladie et du malade, on constate que la mère pense certaines choses de la maladie de son enfant, en l'occurrence que celle-ci n'est pas une maladie physique, mais spirituellement provoquée. En effet, il s'agit d'un jeune qui poursuivait ses études supérieures à l'étranger. C'est lors des vacances d'une année à Lomé que sa maladie a commencé par des malaises sous forme de maux de ventre et plus tard des brûlures d'estomac. Selon le patient, le premier diagnostic était une appendicite. Cependant, d'une appendicite, le jeune homme a connu plusieurs épisodes de cancer en l'espace de quelques années. En 2016, juste avant l'obtention de son master lorsqu'il était en vacances chez ses parents, selon les propos de sa mère et des médicaux, son traitement évoluait bien. Il est entre-temps retourné à l'étranger pour sa soutenance. Cependant, il connaîtra un nouvel épisode. Cette fois une tumeur de l'iléon en mai 2018, peu de temps après son retour au pays. Lorsque nous l'avons rencontré en novembre 2018, il était sous traitement pour une métastase des vertèbres et du poumon et avait une mobilité totalement réduite.

Dans les propos de sa mère, on comprend son désir d'envoyer son enfant loin, dès qu'il se sentira mieux pour s'occuper tout seul de lui-même et continuer son traitement (Quand tout ça va finir par la grâce de Dieu, il faut l'envoyer loin, loin du regard des malveillants). En effet, pour la maman, la maladie de son fils reviendrait à chaque fois que celui-ci rentre au pays. Tant qu'il est ailleurs, soit il se porte bien soit son traitement évolue bien. Pour la 
mère, son fils serait victime d'attaques spirituelles de l'entourage ou de proches parents. En tout cas, il s'agirait de personnes proches de la famille ou qui en font partie et qui ne seraient pas enchantées par "l'évolution ou la réussite »de son enfant, raison pour laquelle elle désire l'envoyer assez loin de leurs regards. Plus loin, elle dira, " je ne veux même plus le voir du tout ici, il ne s'agit pas d'aller et de revenir, parce que quand je ne le vois pas, c'est que je sais qu'il va beaucoup mieux... ».

À l'opposé des premières perceptions répertoriées sur le cancer, celleci n'est pas rattachée à la nature de la maladie, ou ses origines historiques mais plutôt à sa cause. Ainsi, alors que dans le discours médical on parle de plusieurs facteurs de risques, pour ces acteurs rencontrés, le cancer peut avoir une cause bien spécifique, l'envoûtement spirituel de l'homme.

\section{Le cancer, une maladie de la punition ou une épreuve divine}

Cette perception également tente d'établir une cause précise au cancer. Selon des acteurs rencontrés sur le terrain, "...Quand j'étais plus jeune, j'ai pratiqué un avortement... Je ne sais pas s'il y a un lien, mais depuis l'annonce de ma maladie, je n'arrête pas de penser à ce que j'ai fait... (Femme, 45 ans, cancer du sein, février 2019).

"Je sais que cette maladie n'est qu'une épreuve, une mauvaise passe, je sais que mon Dieu est vivant et qu'il me fait juste passer par ce moment comme Job dans la Bible. Et comme Job dans la Bible je m'en sortirai » (Parent d'un jeune souffrant d'une tumeur de l'intestin grêle, novembre 2018).

"Normalement toute faute commise est punie par une épreuve ou par une maladie, ça dépend aussi de la faute. Ce n'est pas une punition divine car Dieu ne punit pas, c'est nous-même qui faisons des bêtises qui nous rendent malades après ». (Personne-ressource de la communauté éwé, août 2018).

Cette perception établit également une cause spécifique au cancer, la punition pour les fautes commises dans la vie, ou l'épreuve. La différence entre cette perception et la précédente est que le premier peut être humainement provoqué et avec des intentions malveillantes et peut être non justifié, alors que celui-ci est plutôt compris comme le fruit de la «volonté de Dieu ». En effet, il peut s'agir d'une punition due à une faute, à un « péché » commis contre Dieu, la nature, la transgression d'une règle ou le non-respect d'un interdit social, culturel ou religieux. Cette perception est souvent rencontrée chez les chrétiens pentecôtistes. Le personnel soignant également en fait parfois cas. L'épreuve du cancer est une épreuve vécue très étroitement avec la question religieuse et de la foi.

Cette perception du cancer est nourrie par certains discours religieux sur les médias et dans les églises, présentant le cancer comme une ultime maladie touchant les pécheurs, la plus mauvaise qu'une personne puisse avoir ou alors à une épreuve divine. Ce discours est couramment véhiculé dans le 
cinéma africain. Nous pouvons citer comme exemple, les films nigérians communément appelé à Lomé ibofilm. ${ }^{45}$ Lorsque la «mauvaise maladie » touche une personne considérée comme exemplaire ou pieuse qui a un cancer, on considère qu'il traverse une épreuve décisive qui s'achèvera par la victoire de sa foi, une délivrance. Cependant, quand il s'agit d'un individu dont la vie n'est pas socialement ou religieusement prise comme exemple, on parle de «punition pour ces péchés ».

\section{Le cancer, une maladie de la civilisation}

Les propos recueillis sur le terrain mettent aussi en cause les différentes formes de changement liées à la civilisation, à la modernisation et aux nouveaux modes de vie.

«Ce sont des maladies causées par l'alimentation. C'est en ville que tout le monde mange tout, mange civilisé, nous ne pouvons même pas être sûrs des choses que nous mangeons, le sucre, le miel sont trafiqués, et nous pensons manger civilisé, les cubes, les conserves et tout ça, des plastiques transformés en riz. Dans la campagne on mangeait paysan et rien de tout ça n'existait » (Personne-ressource kabyè, février 2019).

«Nos grands-parents mangeaient des choses très naturelles et on n'entendait jamais parler de ces maladies, aujourd'hui, plus rien n'est naturel, même notre sol est pourri d'éléments chimiques et toxiques pour nous, même les choses que nous mangeons n'ont plus le même goût qu'avant, même l'air que nous respirons est tellement pollué, il n'est pas surprenant que ces maladies nous envahissent » (Parent d'un patient, cancer de l'estomac, Novembre 2018).

«Il semble aussi que nos téléphones et nos ordinateurs et les appareils électro ménagers sont la cause du cancer, on va faire comment? » (Fille d'une patiente, cancer du sein, février 2019).

La modernisation, le changement climatique, la dégradation de l'environnement, la consommation des conserves, des fast Food, du gras, l'utilisation des pesticides sont souvent revenus au niveau de tous les acteurs rencontrés dans la cause du cancer. Ces éléments sont aussi mis en cause par les organisations de santé au niveau international, surtout lorsqu'elles parlent de la transformation des modes de vie et ou du style de vie. Cette représentation n'établit pas la modernisation et le changement de style de vie comme cause directe au cancer, mais plutôt comme une réalité mondiale qui participe à l'ampleur que prennent le cancer et les autres maladies chroniques ces dernières années.

${ }^{45}$ Comme en anglais films ibo, du nom d'une communautés ethnique nigériane connue pour ses réalisations cinématographiques 


\section{Le cancer, une maladie comme les autres}

Les données recueillies sur le terrain renseignent également sur la considération du cancer comme une maladie comme les autres. "Moi-même je savais que quelque chose n'allait pas et j'avais hâte de savoir ce que c'était. Quand on me l'a annoncé ça ne m'a fait aucun effet. C'est une maladie, on peut la traiter, mais j'ai eu peur de l'argent qu'il va me falloir dépenser. » (Femme, institutrice à la retraite, cancer du sein, août 2016) "Quand on me l'a annoncé, je n'ai pas eu ce choc, avec les analyses qu'on me demandait et les recherches personnelles que moi-même je faisais des recherches sur les termes techniques employés, je savais que ça nous mènerait vers quelque chose de ce genre...Toutes les maladies se soignent, et celle-ci aussi... »(Homme, informaticien, 32 ans, tumeur de l'iléon).

Il n'a pas été très fréquent de rencontrer des acteurs qui considèrent le cancer comme une maladie comme les autres. Même si leur propos est entaché de d'autres perceptions «négatives » de la maladie, notamment la peur le doute et l'incertitude, ce sont des gens qui sont informés et qui s'informent constamment sur la maladie. Ils font l'effort de croire et de se convaincre que c'est une maladie comme les autres pour «mieux espérer s'en sortir». Cependant, il est à relever qu'à l'instar des deux malades cités dans les verbatim précédents, les patients qui considèrent le cancer comme une maladie tout comme les autres, ont un niveau d'instruction supérieur, un statut socioprofessionnel précis et un accès plus facile aux informations sur la maladie. Les verbatim précédents sont ceux d'une institutrice à la retraite. Malgré son âge, celle-ci s'informe beaucoup sur sa maladie. Le second verbatim est celui d'un jeune titulaire d'un master en informatique. D'autres pensant les mêmes choses sont des fonctionnaires. Cependant, en moyenne il faut souligner que tous les malades ou parents de malades qui pensent ainsi ont un niveau d'instruction supérieur.

Cette perception est très répandue chez les soignants, notamment au niveau des médecins spécialistes. Pour le personnel soignant, principalement les acteurs auxiliaires, certains disent que le cancer est une maladie comme toutes les autres. Cependant, on peut également en rencontrer qui ne croient pas en cette conception.

\section{Discussion}

De manière générale dans ce travail, on note des perceptions «négatives » sur le cancer au niveau des différents acteurs rencontrés. L'épreuve du cancer est particulière, individuellement et collectivement vécue. Les perceptions de la maladie chez les patients, leurs proches et les acteurs de soins dans les formations sanitaires à Lomé sont construites d'une part sur la base de la « complexité » autour de la maladie et d'autre part compte tenu des causes que les individus lui attribuent. Les «incertitudes» 
biomédicales autour de la maladie accentuent aussi la méfiance et la peur autour du cancer. L'histoire personnelle des individus et des familles ont une place importante dans cette construction telle que présentée par les résultats de cette recherche.

Comme abordé dans les résultats de travail, la représentation d'une maladie est étroitement liée à l'explication que les individus lui donnent et à la cause qui lui est conférée. Dans le cas du cancer, il est difficile aux malades et à leur entourage de le comprendre parce que la maladie en question n'a pas de cause précise connue (Friard, 2017). Or, l'établissement de la cause d'une maladie facilite sa compréhension et son acceptation par le malade et par son entourage (Laplantine, 1986). Il est donc important pour le malade ainsi que pour sa famille de pouvoir s'expliquer « l'évènement » que constitue « cette » maladie (Augé, 1984 ; Moulin, 2005). Dans son travail sur la définition sociale de la maladie, Kleinman (1980) montre que celle-ci n'a d'explication que dans l'appréhension de la personne qui en parle ou qui la vie. Ainsi, l'auteur distingue la maladie du médecin (desease) et la maladie du malade (illness). La maladie du médecin est la maladie comprise au sens médical du terme. Elle peut être guérie (to cure) par des procédés médicaux. La maladie du malade, quant à elle, est exprimée comme une réalité sociale vécue par l'individu et par sa communauté (illness). Cette forme de maladie n'est jamais guérie au sens médical ou radical du terme. Elle est plutôt soignée (to heal), ainsi le malade pour recouvrer la santé sociale ne se contente pas des soins médicaux, mais va au-delà en faisant objet d'une réparation ou d'une purification de son corps social souillé par la maladie en question. Dans ce cas précis, certaines maladies ne sont jamais réparées au sens définitif du terme. C'est le cas du cancer lorsqu'on dit qu'il n'est jamais guéri ou lorsqu'on parle de rémission. Comme présenté dans les résultats de ce travail, les patients ont du mal à appréhender le cancer, lorsqu'il est dit que malgré la rémission il peut resurgir à tout moment de la vie. L'individu se voit dès lors condamné à ne jamais observer cette réparation ou cette purification sociale. La maladie devient une tache impure, une honte (Gruénais \& Ouatara, 2008). Ce développement cadre avec l'objectif de ce travail d'analyser les perceptions que les malades et leur entourage ont du cancer à Lomé. En effet, une maladie est non seulement un disfonctionnement, un « désordre » biologique mais aussi social (Augé, 1984) et les procédés thérapeutiques qui vont avec doivent prendre en compte cette double dimension. Dotsu (2018) confirme les « ambiguïtés » autour du cancer en pays éwé en disant :

« Le cancer est aussi en pays éwé une maladie aux contours flous. Le cancer est l'une des maladies émergentes les plus inquiétantes à voir son statut en tant que maladie... Il commence par un point et se propage dans tout le corps de l'individu selon la pensée communautaire. Une classification nosographique 
assez dense montre qu'il s'agit d'une maladie qui existait mais très mal connue, dangereuse et surtout très mortelle. » (Dotsu, op. cit. : 219).

Cette incertitude ne se limite pas aux malades des formations sanitaires visitées pour cette étude, ou au pays éwé du Togo. Même dans le discours médical moderne les perceptions du cancer sont empreintes de beaucoup d'incertitudes et de paradoxes (Moulin, 2005). Bretonnière (2016) parle d'espaces de «subjectivités » dans lesquels se rencontrent, se confrontent et se négocient les «subjectivités » des malades et celles des soignants, ce qui est en lien avec l'objectif de cette recherche d'analyser également les perceptions du cancer par les soignants. Comme analysé dans les résultats présentés plus haut, les malades ne sont pas les seuls à ne pas pouvoir appréhender la maladie et à se construire une représentation du cancer. Les soignants construisent également de manière individuelle et parfois collective, leur propre modèle explicatif de la maladie basé sur des constructions tout aussi individuelles, mais liées à leur vécu, à leur histoire et à leur culture d'appartenance.

Comme pour toute pathologie, c'est le rôle principal des soignants, notamment les médecins de donner des explications sur la maladie aux patients, avec les mots qu'il faut les informations sur la maladie aux patients et à leur personnel (Fainzang, 2006). Cependant, dans le cas du cancer les soignants n'ont pas toujours toutes les précisions sur les contours du cancer. C'est ce que souligne Harouna (2001) en ces termes: «Alors, c'est le désarroi, elle qui a toujours cru qu'après tous ces examens son mal serait désormais identifié, se voit aujourd'hui refusée pour l'intervention ou même déclarée non guérie alors que "son mal a été emporté par la chirurgie » (Harouna, op.cit. : 83). Face à ces incertitudes, il arrive que les patients et leur entourage se tournent vers d'autres formes de thérapies dans le but de traiter le cancer (Cohen \& Rossi, 2011).

En pays éwé, les maladies considérées comme ordinaires sont perçues comme normales, mais les maladies persistantes, telles que les maladies chroniques, sont perçues comme ne relevant pas de la biomédecine, mais plutôt fruit d'un dysfonctionnement cosmologique, de malédiction ou de mauvais sors (Atchrimi, 2008). En effet, Dotsu (op.cit. :220) revient sur la perception du cancer comme une maladie spirituellement provoquée. Il dit : "L'une des causes les plus importantes citées est l'envoûtement par les sorciers, les envieux de même que toute personne avec qui l'on est en conflit. Ainsi les causes spirituelles ou surnaturelles de la maladie dans le modèle explicatif des patients et de leur entourage ressorti dans ce travail viennent compléter les imprécisions biomédicales sur les causes du cancer. Pour Sarradon-Eck (2004) : "Dans l'imaginaire populaire, le cancer a toujours été une figure du Diable, dont le comportement sournois, rusé et hypocrite 
ressemble aux différentes bêtes maléfiques auxquelles il a été associé (crabe, araignée, serpent, crapaud (Gros 1994 cité par Sarradon-Eck, op.cit. : 2).

Par ailleurs, selon Dotsu (2018), les populations togolaises pensent que ceux qui souffrent du cancer ne peuvent pas vivre au-delà de six mois. Cette assertion montre la présence quasi incontournable de l'idée de la mort dès que le cancer est évoqué. Ce qui est également abordé dans ce travail, lorsque le cancer est étroitement relié à la fatalité. Cependant, l'image de la mort véhiculée par le cancer n'est pas typique à L'Afrique. Moulin (2005) dit que le cancer est considéré comme une maladie «effrayante ». Au-delà de cela, la mort par cancer n'est pas perçue comme étant une mort ordinaire. Selon Sarradon-Eck (op.cit), dans la représentation populaire du cancer, la mort par cancer est perçue comme une sale mort :

« Le cancer renvoie aussi à l'image paradigmatique de la mauvaise mort : la mort annoncée dans des souffrances qui traînent en longueur, mais aussi la « pourriture » du corps, la «noirceur», la « dévoration » par le mal qui se nourrit de l'intérieur du corps à l'image du crabe toujours présente dans la pensée populaire » (Saillant, 1988 cité par A. Sarradon-Eck, op.cit. : 2)

Comme le présentent les résultats de cette recherche, dans la plupart des cas, pour le malade et pour son entourage (Delvaux, 2006), et pour certains acteurs du monde soignants, le cancer est conçu comme étant une maladie destructrice au sens où l'entend Herzlich (1969). Cependant, le cancer n'est pas considéré comme incurable et inévitablement mortelle seulement au Togo. Soum-Poulayet (2006) dans un travail sur la France, résume cette perception en déclarant que la représentation symbolique du cancer est fortement liée à une mort à grande échelle, incontrôlable et incontrôlée, malgré les progrès médicaux. Moulin (2005), insiste en disant, le cancer est personnifié tantôt comme un ennemi invisible, sournois, silencieux, impitoyable, condamnant ses victimes à une mort quasi certaine.

En outre, en ce qui concerne la perception du cancer comme une maladie de la civilisation et du changement du style alimentaire et des riches relevés par les résultats de cette étude, Dotsu (op.cit.) dans son enquête révèle également que dans l'imaginaire populaire au sud du Togo, le cancer est essentiellement dû à la « mauvaise » alimentation. Les «aliments en boîte de conserve, les huiles de gros bidons qui se solidifient et qu'on met au soleil avant consommation, les aliments mal préparés ou mal traités...Les produits agricoles cultivés avec beaucoup de produits chimiques » sont incriminés (Dotsu, op.cit. :220). Pour appuyer ce point de vue, dans son travail sur les représentations profanes et savantes du cancer en occident, notamment en France, Sarradon-Eck (op.cit.) revient dans la représentation profane sur la conception du cancer comme une conséquence du mode de vie moderne avec la mise en cause de la rupture entre l'individu et la nature. Cette pensée dénonce la nourriture «chimique » chargée de toxines, de pesticides, de 
conservateurs; considérée comme «trafiqués» ou «truquée ». L’auteur ajoute qu'après la nourriture comme précisé par les résultats, les ondes, notamment les transformateurs, les lignes haute tension, les ordinateurs, les téléphones portables et les champs magnétiques sont également indexés (Sarradon-Eck, 2004 : 9). Dans le même ordre d'idées, l'auteur évoque que dans la pensée savante, après le tabac et l'alcool, c'est la nourriture trop « riche » qui est incriminée, notamment les graisses animales, les viandes dans la cause du cancer tout comme dans les maladies cardiovasculaires (SarradonEck, op.cit. :10), une perception similaire à celle des acteurs rencontrés dans le cadre de ce travail, pour qui le cancer est une maladie de « riches ».

\section{Conclusion}

Aux termes de cette recherche, il faut noter que le cancer à Lomé est soumis à plusieurs formes de perceptions et de représentations sociales. Dans leur ensemble, elles sont constituées autour de la négativité, de la peur, de la honte et de la mort assurée. L'incertitude et l'ambiguïté marquent également les perceptions du cancer dans les formations sanitaires de Lomé. Ces constructions sont accentuées par le discours biomédical «imprécis » autour de la maladie. Tout compte fait, tous les acteurs rencontrés ne lient pas le cancer à la fatalité, même si la peur de la maladie est presque toujours présente. En effet, plusieurs soignants, certains patients et des membres de leur entourage rencontrés dans les différentes formations sanitaires considèrent le cancer comme une maladie comme d'autres. La particularité des patients et proches en question, est qu'ils ont un niveau d'instruction relativement élevé et ont accès à d'autres sources d'information sur la maladie. Dans l'ensemble, les causes du cancer, sont très étroitement liées à la civilisation et à la modernisation, notamment à l'alimentation moderne. Cette appréhension est présente auprès de tous les acteurs. Rappelons que le cancer est aussi très étroitement lié au surnaturel et au spirituel dans ses causes, comme une tentative de trouver une explication à la maladie. En définitive, il convient de noter que le cancer est une maladie entourée de complexité et un tel caractère de la pathologie est un paramètre très évocateur qu'il ne faut pas négliger lorsque l'on parle du cancer, quelle que soit la société dont il est question et l'angle sous laquelle la maladie est étudiée.

Malgré les résultats auxquels il est parvenu, ce travail ne peut prétendre avoir recensé de manière exhaustive les perceptions du cancer à Lomé. Les recherches se sont limitées aux acteurs autour des personnes malades de cancer, c'est-à-dire les malades eux-mêmes, les patients, certains membres de leur entourage rencontrés notamment dans les mêmes formations sanitaires et les soignants. De même, la recherche a couvert seulement trois formations sanitaires de la ville. Cette étude gagnerait à être élargie à d'autres couches de la population en vue de faire une possible comparaison entre les perceptions 
des personnes directement touchées par la maladie et d'autres qui ne le sont pas. Par ailleurs, il conviendrait également de pousser les recherches en vue d'analyser les interactions et les relations entre les patients, les membres de leur entourage et les acteurs de soins sur la base des différentes perceptions enregistrées et de mûrir d'autres réflexions mettant en jeu les perceptions du cancer et l'itinéraire thérapeutique des malades.

\section{References:}

1. Amegbor,K., Darre, T., Ayena, K. D., Padaro E., Tengue. K., Abalo. A. and Napo-Koura, G., (2011). Cancers in Togo from 1984 to 2008: Epidemiological \& Pathological Aspects of 5251 Cases». Journal of Cancer Epidemiology, ID 319872, doi: 1155/2011/319872, 1-8.

2. Atchrimi, T., (2008). La problématique de l'action publique au Togo. Étude de cas : la lutte contre le Sida. (Thèse de doctorat, en vue de l'obtention du titre de docteur en Sociologie) Université FrancheComté.

3. Augé, M., (1984). «Ordre biologique, ordre social : la maladie, forme élémentaire de 1'événement », in AUGÉ Marc et Claudine Herzlich (dir.), Le sens du mal : anthropologie, histoire, sociologie de la maladie, (p. 35-92). Paris : Edition Les Archives Contemporaines.

4. Blanchet, A., Ghiglione, R., Massonat, J. \& Trognon, A., (2005). Les techniques d'enquête en sciences sociales. Paris : Dunod.

5. Bonnet, D. \& Jaffré, Y., (2003). Les maladies de passage. Transmissions, préventions, hygiènes en Afrique de l'Ouest. Cahiers d'études africaines, 182, 446-449.

6. Bourdon, B. (2012). Le patient, son diabète, ses représentations : comment amener ce patient vers l'observance optimale $d u$ traitement. Mémoire de l'Institut de Formation en Soins Infirmiers Val de Lys-Artois Saint Venantun.

7. Bretoniere, S., (2016). Autonomie et espace des subjectivités en oncologie. DERBEZ Benjamin, Hamarat Natasia, Marche Hélène (dir). (2016), La dynamique des subjectivités en cancérologie (p. 2040). Toulouse : érès.

8. Canguilhem, G. (1966). Le normal et le pathologique. Paris, PUF.

9. Cohen, P., Rossi, I., (2011). Le pluralisme thérapeutique en mouvement. Anthropologie \& Santé, (2), np. DOI :10.4000/anthropologiesante.606. Disponible en ligne sur http://anthropologiesante.revues.org/606; (Consulté le 27 janvier 2017).

10. Dany, L. (2011). Quand le traitement se fait maladie. Commentaire. Sciences sociales et santé, 29(3), 75-80. DOI : 10.3917/sss.293.0075. 
URL : https://www.cairn.info/revue-sciences-sociales-et-sante-20113-page-75.htm Consulté le 30 mars, 2019.

11. Delvaux N., (2006). L'expérience de cancer pour les familles. Cahiers critiques de thérapie familiale et de pratiques de réseaux, 1(36), 81101, DOI 10.3917/ctf.036.101.

12. Derbez, B., Hamarat, N. \& Marche, H. (2016). La dynamique sociale des subjectivités en cancérologie. Toulouse : Eres.

13. Dotsu, Y. M., (2018). Dynamique de l'éducation sexuelle et santé de la reproduction chez les éwés du Togo. Thèse de doctorat en Anthropologie, Université de Lomé.

14. Fainzang, S. (2006). La relation médecins-malades : information et mensonge. Paris : PUF.

15. Friard, D. (2017). Les représentations de la maladie, Serpsy, disponible en ligne sur http://www.serpsy.org/formation_debat/diagnostic/representations.ht ml\#sommaire, (Consultée le 03 juillet 2017).

16. Gnamien, K. (2018). L'information pour le développement durable de la santé. Cas de l'usage des réseaux sociaux dans la lutte contre le cancer de la prostate en Côte d'Ivoire. European Scientifique Journal 14 (3), 460-476.

17. Gayibor, N. (2011). Histoire des Togolais : Des origines aux années 1960, Tome 1, De l'histoire des origines à l'histoire du peuplement. Lomé : Presses de l'Université de Lomé.

18. Gros, D. (1994). Les seins aux fleurs rouges. Paris : Stock.

19. Gruenais, M.E. \& Ouattara, F. (2008). Des malades comme les autres » ou comment les soignants traitent les malades du sida et la question de la stigmatisation au Burkina Faso. Sciences et technique, Sciences de la santé (1), 93-100.

20. Harouna, Y. (2001). La femme d'Afrique et son chirurgien face au cancer du sein. Médecine d'Afrique Noire, 48 (2), 1.

21. Herzlich, C. (1969). Santé et maladie. Analyse d'une représentation sociale. Paris : Ecole des Hautes Etudes en Sciences Sociales.

22. Jaffré, Y. (1999). La maladie et ses dispositifs. Dans Jaffré Y., Olivier de Sardan J.P. (dir), La construction sociale des maladies (pp.41-68). Paris : PUF.

23. Jodelet, D. (1984). Représentation sociale : Phénomènes, concept et théories. Dans Moscovici S., Psychologie sociale (pp. 357-378). Paris : PUF.

24. Kleinman, A. (1980). Patients and healers in the context of culture. An Exploration of the Borderland between Anthropology, Medicine and Psychiatry. Berkeley: University of California Press. 
25. Kpotchou, K. (2018). Alimentation, réseaux sociaux et peurs émergentes. Rekoss, 2(1), 13-39.

26. Kubler-ross, E. (1976). Les derniers instants de la vie. Paris: Labor et Fides.

27. Lacombe, M., Pradel, J.-L. \& Raynaud, J.J. (2005). Dictionnaire médical à l'usage des iDE. Paris : Lamarre.

28. Laplantine, F. (1986). Anthropologie de la maladie. Paris: Payot.

29. Lebeer, G. (2016). Préface, dans DERBEZ B., HAMARAT N., MARCHE H. (dir), La dynamique des subjectivités en cancérologie (pp.7-11). Toulouse : Érès.

30. Moulin, P. (2005). Imaginaire social et cancer. Revue Francophone Psycho-oncologie, 4 (4), 261-267.

31. Organisation Mondiale de la Santé, (2014). Profils des pays pour le cancer. Disponible sur https://www.who.int/cancer/countryprofiles/fr/ (consulté le 22 octobre 2015).

32. Organisation Mondiale de la Santé, (2015). Maladies Non Transmissibles/ Maladies chroniques, disponible sur http://www.who.int/topics/noncommunicable diseases/fr/2015.

33. Ourobitasse, E. (2015). Les stratégies d'intégration des communautés étrangères à Lomé et la problématique de la cohésion sociale: regards croisés sur les communautés Haoussa et Ibo. Thèse de doctorat en sociologie, Université de Lomé.

34. Saillant, F. (1988). Cancer et culture. Produire le sens de la maladie. Montréal : Editions Saint-Martin.

35. Sarradon-Eck, A. (2004). Pour une anthropologie clinique : saisir le sens de l'expérience du cancer, in Ben Soussan P. (dir.), Le cancer, approche psycho dynamique chez l'adulte (pp.31-45). Toulouse : ERES.

36. Schwartz, B. (2011). La relation de confiance soigné-soignant. Travail de fin de formation en Soins Infirmiers. Lieu ? pays ?

37. Soum-Poulayet, F. (2006). Le risque émotionnel en cancérologie. Problématiques de la communication dans les rapports entre soignants et soignés. Face à face, 2006 (8) disponible en ligne sur http://faceaface.revues.org/257 (Consulté le 15 février 2017). 